\title{
Avaliação da qualidade do Programa Saúde da Família em municípios do Ceará. Uma abordagem multidimensional
}

\author{
Evaluation of the quality of Family Healthcare Program \\ in municipalities of Ceará. A multidimensional approach
}

Mauro Serapioni ${ }^{1}$

Marcelo Gurgel Carlos da Silva ${ }^{2}$

${ }^{1}$ Centro de Estudos Sociais, Universidade de Coimbra. Colégio de S. Jerónimo, apartado 3087. 3001-401 Coimbra Portugal. mauroserapioni@ces.uc.pt ${ }^{2}$ Centro de Ciências da Saúde, Universidade Estadual do Ceará. Av. Paranjana, 1700, Itaperi. 60740-000 Fortaleza CE
Abstract We evaluated the quality of the Family Healthcare Program of Ceará (Brazil) by adopting a multidimensional model based on the theoretical assumptions of two important scholars, both well-known at an international level, namely Avedis Donadedian and John Øvretveit. We adopted the well-known approaches to the quality of healthcare of the first author: structure, process and outcome. Of the latter, we adopted the three dimensions of quality: quality perceived by patients, by professionals and by managers. The research was conductedten municipalities of the State of Ceará. Even though different nuances and emphases were used, the informants (physicians, nurses, coordinators of family healthcare teams, municipal secretaries of health and users) some aspects responsible for the improvement of the quality of Family Healthcare were identified. As a final result the study highlight a range of dimensions and quality indicators according to the perspective of the different actors involved in the study. Key words Evaluation of Healthcare Quality, Family Healthcare Program, Patient satisfaction, Brazil
Resumo Com o intuito de responder às necessidades dos sistemas locais de saúde, este estudo avaliou a qualidade da atenção das equipes do Programa Saúde da Família (PSF) em Municípios do Ceará (Brasil) adotando um modelo de análise multidimensional fundamentado nas elaborações teórico-metodológicas de dois importantes autores conhecidos em nivel internacional: Avedis Donadedian e John Øvretveit. Do primeiro autor, foram utilizadas as três abordagens da qualidade, ou seja, a estrutura, o processo e os resultados. Do segundo autor, foram adotadas as três dimensões da qualidade: qualidade percebida pelos pacientes, qualidade técnica definida pelos profissionais e qualidade na perspectiva gerencial. $O$ estudo foi realizado em dez municípios do Estado. Embora com diferentes nuances e ênfase, os informantes entrevistados (médicos e enfermeiros das equipes, coordenadores do PSF, secretários de saúde e usuários) identificaram um conjunto de aspectos referentes à estrutura e ao processo do serviço, que deveria qualificar o PSF. Como resultado final do estudo, os pesquisadores apresentam um conjunto de dimensões e indicadores de qualidade, segundo a perspectiva dos diversos atores envolvidos.

Palavras-chave Avaliação da qualidade em saúde, Saúde da Família, Satisfação dos pacientes, Brasil 


\section{Introdução}

Desenvolver programas de garantia da qualidade nos serviços de saúde tem sido uma das principais preocupações da Organização Mundial da Saúde (OMS), a partir do início da década dos anos $80^{1}$. Contudo, na prática, foi se consolidando uma maior preocupação por considerações referentes a custos e recursos dos serviços de saúde, que puseram em primeiro plano a avaliação custo-efetividade ${ }^{2}$.

Sucessivamente, como consequência de múltiplas atividades de informação e divulgação, o discurso sobre qualidade da atenção à saúde voltou a ser prioritário na agenda das organizações internacionais e dos governos nacionais. De fato, nos últimos anos a qualidade é considerada um componente fundamental na maioria dos países do mundo, independente do nível de desenvolvimento econômico e do tipo de sistema de saúde adotado ${ }^{3}$.

Apesar do crescente interesse dos últimos anos, a qualidade representa um desafio para a maioria dos atores que opera na arena da saúde que ainda não chegou a um acordo sobre uma definição operacional apropriada e compartilhada. A qualidade em saúde tem muitas facetas e autores diferentes podem utilizar significados distintos para esse termo $0^{4,5}$.

Analisando a literatura sobre o tema é possível encontrar inúmeras abordagens e definições de qualidade. Sem dúvida, o conceito de qualidade não é simples, nem unívoco, mas complexo e polivalente ${ }^{6}$. A complexidade da qualidade depende de vários fatores: a) em primeiro lugar, é preciso assinalar a complexidade da mesma concepção de saúde que, após as críticas ao processo de medicalização da vida ${ }^{7-9}$, considera a pessoa na sua unidade e integridade biopsíquica, inclusos os componentes espirituais, relacionais e sociais; b) essa complexidade é reforçada pela variedade de atores que agem nos sistemas de saúde; c) a pluralidade de abordagens e metodologias de análise da qualidade depende, também, do caráter intrinsecamente multidimensional do conceito de qualidade ${ }^{6,10-12} ;$ d) é preciso também focar as peculiaridades das ações de saúde que não produzem bens, mas serviços que incluem um amplo componente de trabalho imaterial com acentuada personalização e relacionalidade ${ }^{13}$; e) finalmente, a concepção de qualidade é condicionada por fatores de contexto históricos, culturais, políticos, sociais e institucionais.

"De cultura a cultura - ressalta Donabedian ${ }^{14}$ mudam as normas de adequação e a disponibili- dade dos recursos, as preferências das pessoas, suas crenças e, consequentemente, devem mudar os critérios para avaliar a qualidade da atenção". Todos esses fatores de complexidade enriqueceram o conceito de qualidade da atenção à saúde, mas, ao mesmo tempo, o tornaram de difícil definição.

A mesma situação e as mesmas dificuldades encontram-se no âmbito e no processo de implementação da atenção primária, onde a qualidade do atendimento não tem recebido a necessária importância e prioridade. A partir dessa constatação, a OMS lançou, em 1989, um programa de promoção da avaliação da qualidade da atenção primária, com o objetivo de sensibilizar gestores, administradores e profissionais da saúde e desenvolver métodos e competências apropriadas ${ }^{3}$. Ao ignorar o tema da qualidade que afeta a maioria da população, se consolidam, segundo a OMS, as condições de desigualdade, já que a qualidade torna-se uma prerrogativa de uma pequena minoria da população ${ }^{3}$.

Todas estas questões colocam em primeiro plano a necessidade de realizar atividades de avaliação da qualidade da Atenção Primária. O tema da satisfação dos usuários foi bastante explorado nos últimos 15 anos, mas, ainda são poucas as avaliações da qualidade mais abrangentes, que consideram a participação de diferentes atores envolvidos na produção da saúde. De fato, é fundamental desenvolver uma nova metodologia de avaliação adequada às características desse programa, que não se limite à análise de custos das atividades empreendidas, nem ao nível de satisfação dos pacientes. No entanto, esta deve reconhecer o pluralismo de valores e favorecer a interação de demandas, experiências e pontos de vistas dos diferentes grupos de interesses ${ }^{15}$. É, outrossim, importante adotar todas as precauções metodológicas e operacionais para que os resultados sejam efetivamente utilizados ${ }^{16}$. Nesse sentido, as avaliações em serviços ou programas de saúde devem subsidiar os processos de decisão, servir de suporte para o (re)direcionamento das práticas profissionais e devem melhorar uma intervenção em seu decorrer, caracterizando-se, em última análise, como ferramenta de gestão ${ }^{17,18}$.

Esse estudo, realizado em 2003, avalia a qualidade da atenção das equipes de Saúde da Família do Ceará, adotando um modelo de análise multidimensional fundamentado nas elaborações teórico-metodológicas de dois importantes autores conhecidos em nível internacional: Avedis Donadedian ${ }^{19,20}$ e John Øvretveit ${ }^{21,22}$.

Do primeiro autor foram utilizadas as três abordagens da qualidade, ou seja, a estrutura, o 
processo e os resultados, que permitiram examinar atentamente os recursos disponíveis, os processos organizacionais, as atividades realizadas e os níveis de satisfação dos usuários. Do segundo autor, foram adotadas as três dimensões da qualidade: qualidade percebida pelos pacientes, qualidade técnica definida pelos profissionais e qualidade na perspectiva gerencial.

Nenhuma dessas dimensões pode ser ignorada, mas, como têm demonstrado várias experiências, os diferentes grupos de interesse (dos pacientes, de seus familiares, dos profissionais e dos gestores) tendem a privilegiar um aspecto ou outro da qualidade com a óbvia consequência de desenvolver abordagens e métodos de avaliação diferentes. A abordagem da qualidade, acrescenta $\emptyset$ vretveit ${ }^{21}$, "não é uma coisa mágica, como, às vezes, foi apresentada, mas um conjunto de métodos e uma filosofia", que obriga as pessoas a enfrentarem conflitos de interesses, a lidarem com situações em que o aumento de um aspecto da qualidade pode prejudicar outro e a elaborarem soluções qualificadas para tais conflitos. Trata-se, obviamente, de um modelo de avaliação que tem mostrado boas potencialidades para abordar a complexidade do fenômeno da qualidade da atenção primária, podendo ser ulteriormente aprimorado em outras investigações. Neste artigo, após ter descrito o contexto e a metodologia do estudo se apresentam os resultados da investigação avaliativa e, sucessivamente, se desenvolve uma análise comparativa das diferentes visões de qualidade.

\section{Contexto do estudo}

Em 1994, o Ministério da Saúde lançou uma proposta de implantação de um extenso Programa Saúde da Família (PSF), resgatando a rica experiência dos Agentes Comunitários de Saúde (PACS). No Estado do Ceará, houve uma ampla adesão a este programa, assim como tinha sido abrangente e pioneira a experiência dos PACS, que deu uma significativa contribuição para o desenvolvimento do programa nacionalmente ${ }^{23}$.

Sem dúvida, a implantação do PSF tem implicado uma apreciável ampliação do acesso da população aos serviços de saúde e um significativo incremento das atividades preventivas em favor dos grupos sociais que estão em maior risco de adoecer e morrer. Porém, ainda existem - segundo vários autores ${ }^{24-26}$ - alguns aspectos críticos no que concerne à avaliação do impacto do PSF, seja em relação ao seu resultado sobre o desempenho do sistema de saúde, seja como estratégia para reduzir as desigualdades e melhorar a situação de sanitária da população.

Nos últimos anos, o Ministério da Saúde tem desenvolvido diversas ações com vista à institucionalização da avaliação da atenção básica ${ }^{27-29}$. Diversos pesquisadores também têm se preocupado em analisar o Programa Saúde da Família. Muitos estudos têm avaliado, dentro dos Estudos de Linha de Base do Proesf, o grau de implantação do PSF ou de determinados componentes do mesmo (saúde reprodutiva; atividades de prevenção AIDS/DST; integralidade da atenção; atenção integrada às doenças prevalentes da infância; cuidados em saúde prestados às gestantes; etc) ${ }^{30-35}$.

Entretanto, o problema da qualidade dos serviços prestados à comunidade ainda representa um aspecto crítico. Isso pode ser evidenciado claramente quando se considera que diversas equipes têm atuado sem a presença do médico ou que a maioria das equipes atende uma população muito maior do que a que foi preconizada pelo Ministério da Saúde. Um outro fator muito importante é o fato de haver um rodízio muito intenso dos profissionais de nível superior em busca de melhores condições de trabalho, prejudicando o seguimento dos pacientes pelo mesmo profissional.

Os estudos avaliativos conduzidos no âmbito do Ministério da Saúde 27,36,37 tinham já apontado a existência de uma grande variedade de modelos de PSF nos municípios do país. Esta constatação reflete também a situação dos municípios do Ceará que apresentam diferentes níveis de organização e funcionamento das equipes do Programa Saúde da Família. Por tratar-se de um programa do Ministério da Saúde que almeja se consolidar como um novo modelo assistencial para o país, consubstanciado no êxito de programas similares em outros países, desenvolvidos ou em desenvolvimento, torna-se imperativo que, "pari passu" a sua consolidação, deva ser instituído mecanismos adequados de avaliação ${ }^{29,38,39}$.

De acordo com as diversas experiências internacionais e nacionais a vertente da qualidade deve ser consagrada como elemento-chave do processo avaliativo. A não implementação de tais procedimentos poderá levar o sistema de saúde a incorrer em erros ou falhas que, por não serem perceptíveis no tempo oportuno, impossibilite a revisão e os ajustes cabíveis para o bom desenrolar do PSF no Ceará. 


\section{Metodologia do estudo}

Esta investigação caracteriza-se, de acordo com as definições de Contandriopoulos ${ }^{40}$, como uma Pesquisa Avaliativa e constitui-se em um estudo de casos múltiplos com níveis de análise imbri$\operatorname{cados}^{41}$. O estudo de caso utilizou uma abordagem metodológica quanti-qualitativa e diversos recursos e técnicas para a coleta, o processamento e análise de dados. A pesquisa foi articulada em diferentes etapas. Na primeira fase foi realizado um workshop com especialistas do PSF (acadêmicos, gestores e profissionais) e com representantes dos usuários (membros do Conselho Estadual de Saúde e de Conselhos Municipais de Saúde). Durante o workshop se discutiu da qualidade das atividades do PSF e dos fatores que a determinam. Pretendia-se, em outras palavras, esboçar uma definição de qualidade da atenção primária adequada ao contexto econômico e social do Estado do Ceará e que pudesse servir como referencia durante o processo de avaliação do PSF.

Posteriormente, após o workshop, para a amostra intencional de casos foram selecionados dez municípios das três macrorregiões de saúde do Ceará (Fortaleza, Sobral e Cariri), a partir dos seguintes critérios: municípios com mais de cinco anos de vigência ininterrupta do Programa Saúde da Família, dentre esses, cinco municípios de pequeno porte (até 20 mil habitantes), quatro municípios de médio porte (de 20 mil até 100 mil) e um município de grande porte (acima de $100 \mathrm{mil}$ ).

Vale ressaltar que o número de equipes estudadas $(n=17)$ foi distribuído de acordo com o porte de cada município, ou seja, pequeno porte (uma equipe), médio porte (duas equipes) e grande porte (quatro equipes). A pesquisa foi realizada nas seguintes seis microrregiões de saúde: Baixo Jaguaribe, Baturité, Brejo Santo, Litoral de Aracati, Maracanaú e Sertão de Crateús. Utilizando os critérios acima listados foram selecionados os municípios e as equipes de PSF. Para a coleta de dados foram utilizadas as seguintes técnicas:

a) Entrevista semi-estruturada com os secretários municipais de saúde $(\mathrm{n}=10)$ e os coordenadores do PSF ( $n=10)$, com a finalidade de: $\mathrm{i}$ ) aprofundar os aspectos relacionados à estrutura, aos recursos materiais e humanos e à organização das equipes e do trabalho; ii) obter a visão deles sobre o que define a qualidade do PSF.

b) Entrevista semiestruturada com os profissionais de nível superior (17 médicos e 17 enfermeiros) que na época da pesquisa compunham as equipes do PSF, indagando sobre: i) a organização, o funcionamento do PSF e o desenvolvimento de suas atividades; ii) as principais dificuldades enfrentadas e os aspectos mais críticos do serviço; iii) as opiniões deles sobre os principais componentes que determinam a qualidade da atenção do PSF.

c) Durante a condução das entrevistas semiestruturadas, foram realizadas observações diretas das unidades $(n=5)$ e dos pontos de apoio $(\mathrm{n}=5)$, com a finalidade de verificar as condições estruturais em que são desenvolvidas as atividades da equipe. Para esse fim, foi utilizado um check-list previamente elaborado.

d) Aplicação de um questionário com 340 pacientes ( 20 por cada equipe) com a finalidade de: a) avaliar o nível de satisfação a respeito dos vários serviços prestados pelo PSF; b) identificar os indicadores da qualidade da atenção na perspectiva dos respondentes. O questionário foi aplicado com os pacientes que se encontravam na sede ou nos pontos de apoio do PSF. Foram entrevistadas apenas as pessoas adultas, com prévio consentimento livre e esclarecido.

Para examinar o material qualitativo advindo das entrevistas semiestruturadas, foi aplicada a análise temática do conteúdo, após a transcrição integral dos relatos dos informantes ${ }^{42}$. Cabe sublinhar que este tipo de análise requer a aplicação de procedimentos técnicos bastante precisos e suficientemente elaborados, para evitar que as interpretações dos relatos das entrevistas sejam influenciadas pelos valores e representações do investigador ${ }^{43}$. No que se refere aos questionários, os dados coletados foram processados e analisados através do sistema EPI-INFO.

Este estudo foi aprovado pelo Comité de Ética em Pesquisa da Universidade Estadual do Ceará. Após esclarecimento, os informantes que aceitaram de participar da pesquisa assinaram o Termo de Consentimento Livre Esclarecido.

\section{Resultados}

Os resultados da pesquisa estão organizados em três seções. Na primeira seção, de acordo com o referencial teórico adotado, se apresentam as opiniões das diversas categorias de informantes (especialistas; profissionais; gestores e gerentes; usuários) sobre os aspectos estruturais e organizacionais do serviço, ressaltando os pontos fortes e as potencialidades do PSF, assim como os pontos críticos que precisam ser aprimorados. Na segunda seção é analisada a experiência dos pacientes com o PSF, bem como os níveis de sa- 
tisfação. Na terceira seção são apresentadas as dimensões e os indicadores de qualidade dos serviços de Saúde da Família, nas perspectivas dos diferentes atores envolvidos, buscando identificar áreas de consenso ou de conflito entre as diferentes visões da qualidade do PSF (Quadro 1).

Finalmente, é preciso sublinhar que essa avaliação centrou-se na estrutura e nos processos de trabalho do PSF, preocupando-se em analisar a disponibilidade de recursos (input), as modalidades organizacionais (process) e as atividades realizadas (output). No que diz respeito aos resultados (outcome) - outro importante componente da abordagem de Donabedian, além da estrutura e do processo - concentrou-se na análise do grau de satisfação dos usuários, sem pretender abordar os resultados em termo de mudanças no estado de saúde da população ou de mudanças de comportamento dos pacientes, que precisariam de estudos muito mais complexos e, obviamente, de muito mais recursos. Porém, cabe ressaltar que a satisfação do paciente é considerada, entre os estudiosos do setor, um dos resultados a ser atingidos pelos serviços de saúde, embora não seja o único ${ }^{44-47}$.

\section{Potencialidades e aspectos críticos do PSF}

Embora com diferentes nuances e ênfase, os informantes entrevistados (médicos e enfermeiros das equipes, coordenadores do PSF e secretários de saúde) identificaram um conjunto de aspectos que, na opinião deles, deveria qualificar o Programa Saúde da Família e que, de acordo com a classificação de Donabedian ${ }^{19,20}$, podem ser agregados nas dimensões da estrutura e do processo da qualidade da atenção à saúde.

\section{A abordagem estrutural da qualidade do PSF}

Há um consenso sobre a necessidade de melhorar a estrutura física e o ambiente de traba- lho, sobretudo nos pontos de apoio, onde a realização de algumas atividades - segundo os depoimentos dos informantes - "pode ser prejudicada", assim como "a privacidade do usuário durante a consulta”. Os profissionais foram mais incisivos em apontar as dificuldades de trabalhar dentro de uma estrutura não sempre adequada às necessidades do serviço e nem sempre dotada de um equipamento mínimo. No que diz respeito ao transporte e aos medicamentos, o estudo registrou uma diferença de análise entre o grupo dos gestores e gerentes, de uma parte, e o grupo dos profissionais, da outra. Em relação ao transporte, a maioria do primeiro grupo não identificou particulares problemas, ao invés dos informantes do segundo grupo que consideraram inadequado o número de carros para o bom desenvolvimento do trabalho das equipes. Essa dificuldade pode chegar a prejudicar as visitas domiciliares. Falando de medicamentos, os profissionais foram mais críticos dos gestores em focalizar a insuficiência da farmácia básica para satisfazer a demanda da comunidade.

Referente à capacitação, houve uma convergência de opinião entre gestores e profissionais a respeito dos investimentos efetuados nessa área pelos municípios, sobretudo a favor dos profissionais de nível superior. Porém, é interessante ressaltar a preocupação dos gestores no sentido de estimular uma maior participação dos médicos em cursos de atualização e a recomendação dos profissionais para promover iniciativas de capacitação focadas na relação com os pacientes e a comunidade.

Finalizando esta parte referente aos aspectos da dimensão estrutural mencionados pelos informantes, cabe enfatizar a preocupação dos secretários de saúde quanto à questão da contratação e rotatividade dos profissionais, tema abordado também pelos especialistas de PSF entrevistados.

Quadro 1. Modelo utilizado pela avaliação da qualidade do PSF

\begin{tabular}{|l|l|l|l|}
\hline \multicolumn{1}{|c|}{ Dimensões da qualidade } & Estrutura & Processo & Resultado \\
\hline Qualidade na visão dos pacientes & & & \\
\hline Qualidade na visão dos profissionais & & & \\
\hline Qualidade na visão dos gestores e coordenadores & & & \\
\hline Qualidade na visão dos especialistas do PSF & & & \\
\hline
\end{tabular}




\section{A abordagem processual da qualidade do PSF}

De acordo com os depoimentos de profissionais, coordenadores e secretários de saúde existe uma práxis gerencial e organizacional bastante consolidada no âmbito das equipes de PSF. Em todas as unidades envolvidas no estudo, cada equipe, de diferente forma e com diversas modalidades, desenvolve atividades de planejamento e avaliação. $\mathrm{O}$ atendimento aos pacientes é organizado de forma espontânea, ou seja, por ordem de chegada dos pacientes. Somente para as pessoas que necessitam de acompanhamento constante, como hipertensos e diabéticos, existe a possibilidade de agendar as consultas.

Em relação ao processo de trabalho, é preciso assinalar a dificuldade dos profissionais de realizar as atividades externas à unidade de saúde, em virtude da grande demanda de assistência ambulatorial e - como relataram os mesmos informantes - porque "na comunidade é ainda predominante o interesse pela consulta e pelo curativo". Assim, as visitas domiciliares e as atividades de promoção da saúde, duas importantes linhas de intervenção do PSF, ficam seriamente prejudicada. A limitada participação dos usuários às atividades preventivas tem sido destacada também no estudo de Ronzani e Silva ${ }^{48}$, realizado em dois municípios de Minas Gerais.

A necessidade de aprimorar as ações de educação e promoção é compartilhada também pelos coordenadores e secretários de saúde que indicam algumas áreas estratégicas a serem priorizadas: saúde mental, gravidez na adolescência, educação sexual etc. Além disso, recomendam aos profissionais fortalecer a relação com a população, estabelecendo parceria com lideranças e conselhos locais. Finalmente, é importante ressaltar a satisfação profissional manifestada pela maioria de médicos e enfermeiros, não obstante as diversas dificuldades que enfrentam no dia-a-dia. Essa satisfação é resultante da valorização, por parte da população, do serviço prestado.

\section{A satisfação dos pacientes}

Captar a opinião dos usuários acerca do que determina a qualidade da atenção à saúde do PSF certamente não foi uma tarefa fácil. Teve-se que enfrentar todas as dificuldades relacionadas à pesquisa de satisfação dos usuários, que a literatura internacional sobre o tema já tinha identificado. Está-se falando daquele fenômeno que alguns estudiosos definem como 'Paradoxo da sa- tisfação ${ }^{49-51}$, ou seja, o fato que, apesar dos serviços de saúde apresentarem vários problemas estruturais, organizacionais e de acesso, as pesquisas continuam levantando um elevado grau de satisfação dos pacientes. Isso pode ser o resultado de variáveis sócio-econômicas e educativas que caracterizam uma significativa parte da população cearense e que podem influenciar a capacidade de assumir um papel ativo. Além disso, diversos estudiosos assinalam também a existência de vários viéses metodológicos que explicam o alto nível de satisfação ${ }^{52-54}$.

Quem tem trabalhado seriamente na tentativa de mensurar a satisfação dos usuários conhece muito bem as dificuldades metodológicas relacionadas com este tipo de pesquisa. No caso desta pesquisa, por exemplo, o questionário mostrou algumas 'insatisfação' para coletar a visão dos usuários sobre o que determina a qualidade do atendimento. Seguramente uma técnica de pesquisa qualitativa (entrevista ou grupo focal) e menos intrusiva $a^{55}$, teria sido mais proveitosa para alcançar este objetivo, embora permitisse abranger apenas um pequeno número de usuário do PSF.

Em coerência com os resultados de outras pesquisas efetuadas, nacional e internacionalmente, com questionários estruturados, o grau de satisfação dos pacientes do PSF envolvidos neste estudo resultou muito alto. Cerca de $85 \%$ dos respondentes avaliaram positivamente $o$ atendimento recebido e mais do $90 \%$ mostraram-se satisfeitos com o relacionamento estabelecido com enfermeiros e médicos. Estes dados devem ser interpretados à luz do perfil dos respondentes, do contexto sócio-econômico do Ceará e da "influência da cultura política e dos filtros de mediação" ${ }^{\text {"50, }}$ que, de alguma forma, condicionam as expectativas e o nível de satisfação dos pacientes. Para pessoas que vivem no interior do Estado, acostumadas a uma escassa cobertura assistencial, o acesso ao serviço de saúde da família com a possibilidade de serem atendidos por médicos e enfermeiros induz, sem dúvida, uma sensação de satisfação $0^{45}$, embora existam - como objetivamente observaram profissionais, gerentes e gestores - alguns aspectos críticos no nível da estrutura e do processo do PSF que podem prejudicar a qualidade da atenção.

\section{Dimensões e indicadores de qualidade na visão dos diferentes atores}

Outro objetivo deste estudo era conhecer a opinião dos diferentes atores sobre os aspectos 
que determinam a qualidade do atendimento do PSF. A seguir, são apresentadas as análises do discurso e alguns depoimentos dos diferentes informantes que responderam sobre os principais fatores que definem a qualidade.

\section{Qualidade na perspectiva dos profissionais}

Em ordem de importância os profissionais apontaram os seguintes aspectos que deveriam ser adequadamente trabalhados para poder consolidar a qualidade desse importante serviço de atenção primária.

a) A maioria dos profissionais entrevistados identificou como um dos elementos definidores de qualidade a relação profissional-paciente, onde se faz necessário estabelecer um vínculo de confiança entre usuário e profissional que favoreça a inserção da equipe no ambiente de vida do paciente, de modo que a mesma torne-se uma conhecedora da "realidade social e cultural de toda a família".

b) Muitos profissionais apontaram, também, a estrutura física como um dos elementos definidores de qualidade. Segundo os mesmos, para que as equipes de PSF desempenhem suas funções de maneira eficiente é necessário que exista "um ambiente físico adequado, que propicie a realização das atividades com conforto e privacidade", atendendo, dessa forma, "tanto as necessidades dos profissionais quanto as dos usuários”.

c) É importante ainda salientar que uma parte significativa dos profissionais pesquisados percebe a questão da educação em saúde (palestras e trabalhos em grupo), como um dos pontos que exige uma melhor implementação. "A grande demanda de atendimento ambulatorial e de tratamento farmacológico - assinalam os informantes - tem dificultado de aprimorar o trabalho educativo junto aos diversos grupos de pacientes".

d) Outro determinante de qualidade, apontado por grande parte dos profissionais, diz respeito ao nível de capacitação dos profissionais que compõem a equipe do Programa. Para os profissionais, se as equipes tiverem uma boa qualificação que possibilite seguir "os preceitos estabelecidos para o Programa Saúde da Família, certamente essas equipes terão condições de desenvolverem um trabalho com qualidade".

e) A necessidade de um maior número de profissionais também surgiu nos discursos dos médicos e enfermeiros. Segundo eles, a insuficiência de profissionais ocasiona, muitas vezes, uma queda no nível de qualidade do atendimento, visto que "as equipes têm que atender uma quantida- de de famílias bem superior ao que preconiza o Ministério da Saúde".

\section{Qualidade na perspectiva de gerentes e gestores}

Respondendo à questão acerca dos principais pontos que determinariam a qualidade no âmbito do PSF, a maioria dos entrevistados destacou, em ordem de importância, os seguintes aspectos como definidores de qualidade:

a) No que se refere ao compromisso profissional, os gestores ressaltam a importância de se ter profissionais altamente dedicados ("que vistam a camisa do PSF, que saibam o que é o PSF”) e envolvidos com "os problemas da comunidade". Isso facilitaria, segundo os informantes, o bom desenvolvimento de ações mais efetivas e adequadas para resolver os problemas de saúde da população.

b) Quanto à atenção dispensada ao paciente, por parte dos profissionais, a maioria dos entrevistados enfatiza que esse é um ponto fundamental para se dimensionar qualidade do atendimento. Para eles, é através da "disponibilidade" e da "sensibilidade do profissional" que se estabelece a relação de confiança entre profissional e usuário, visto que, muitas vezes, segundo os gestores, o usuário procura o atendimento "como forma de ser escutado e de expressar seus problemas" que nem sempre são referentes ao próprio estado de saúde.

c) Outro ponto que, segundo os gestores e gerentes pesquisados, necessita de implementação é a estrutura física. Na opinião dos entrevistados, a existência de um espaço físico "aconchegante" ("um local onde a comunidade se sentisse como se estivesse em casa") e de uma "infraestrutura adequada" promoveria o bom andamento das ações na Unidade de Saúde, possibilitando assim que o atendimento seja definido de qualidade. Os gestores ressaltam ainda seus esforços no sentido de buscar soluções para essa questão do espaço físico.

d) Diversos gerentes percebem também a necessidade de se trabalhar melhor a "educação da comunidade", visando conscientizá-la acerca dos preceitos do PSF, levando assim, a uma redução da "demanda por atendimento ambulatorial" e, por conseguinte, a uma maior disponibilidade das equipes para realizar atividades de prevenção e promoção à saúde nas diversas micro-áreas de atuação da equipe.

e) Finalmente, alguns gerentes acreditam que o nível de atendimento depende de cada equipe, 
estando, portanto, relacionado à formação do profissional. De fato, a qualidade dessa formação pode influenciar seja "a forma de organizar o trabalho da equipe" e seja "a relação com a comunidade".

\section{Qualidade na visão dos pacientes}

Para apreender o pensamento dos usuários, acerca dos principais pontos que para eles definiriam qualidade, lançaram-se as seguintes duas questões abertas: a) O que mais valorizam do PSF? b) O que consideram negativo do PSF? Em relação à primeira pergunta, obtiveram-se 293 respostas de 340 questionários aplicados, ou seja, 86\% dos entrevistados expressaram seu parecer sobre o que determina a qualidade do PSF (Tabela1).

Cerca de $43 \%$ dos que responderam apontaram a boa relação com os profissionais como elemento indispensável para a garantia da qualidade. Nesse sentido, os informantes destacaram as habilidades de comunicação e de escuta dos profissionais; as explicações e informações ofertadas sobre os problemas dos pacientes; a atenção e a paciência dos profissionais; o bom tratamento recebido e o respeito pelo paciente; a capacidade de resolver os problemas; a educação, a gentileza e a simpatia etc. É interessante destacar que $28 \%$ dos informantes manifestaram uma visão muito otimista do serviço, expressando sua grande satisfação com frases de admiração e elogio do PSF e de seus profissionais, tais como: "Eles são ótimos"; "Gosto de tudo"; "Eles aqui são muito legais"; "Eles são gente fina”. É também importante focar que mais de $9 \%$ dos que responderam às perguntas abertas sobre determinantes da qualidade, valorizaram o simples fato de ser atendido pelo serviço de PSF, sem nem sempre se deter na análise da qualidade do atendimento recebido, como se pode observar nos de-

Tabela 1. O que mais os usuários valorizam do PSF

\begin{tabular}{lcc}
\hline \multicolumn{1}{c}{ Itens da qualidade } & $\begin{array}{c}\text { N. de usuários } \\
\text { que responderam }\end{array}$ & Porcentagem \\
\hline Boa relação com os profissionais & 126 & 43,0 \\
Tudo é bom & 83 & 28,3 \\
Ser atendido & 27 & 9,2 \\
A boa atitude do médico & 22 & 7,5 \\
Disponibilidade de remédios & 17 & 5,8 \\
Outras respostas & 18 & 6,2 \\
Total & $\mathbf{2 9 3}$ & $\mathbf{1 0 0}$ \\
\hline
\end{tabular}

poimentos dos informantes a seguir: "Porque não vai ninguém para casa sem ser atendido"; "Porque elas atendem, não botam obstáculos"; "Todas as vezes que eu venho eu consigo, mesmo sem estar marcado"; “Às vezes não tem ficha, mais eles atendem a gente". Estes testemunhos evidenciam quanto é importante para a população de baixa renda poder ter acesso à atenção básica, sobretudo naquelas localidades onde antes do PSF não havia outra opção diagnóstica e terapêutica, além das atividades preventivas desenvolvidas pelos agentes comunitários de saúde. Cerca de $8 \%$ das respostas enfatizam a figura do médico do PSF, destacando suas características profissionais e pessoais, tais como a "atenção", o "tratamento", o “jeito de atender", a "forma de falar" etc. Este dado, embora não muito relevante do ponto de vista da quantidade, parece ser interessante pelo fato de que saiu espontaneamente das falas dos usuários sem que fosse objeto de uma específica pergunta sobre o assunto. Finalmente, é preciso registrar ainda algumas respostas (cerca de 6\%) que valorizam os remédios como um dos componentes da qualidade do PSF, conforme relatam os usuários que responderam ao questionário: "eles passam remédio"; "atendem diretinho e passam o medicamento"; “o pessoal dá o remédio sem fazer cara feia”.

Em relação à segunda pergunta referente aos aspectos negativos do PSF, o que mais chama a atenção é o fato de que somente $14,7 \%$ dos entrevistados (50 usuários de 340) identificaram aspectos problemáticos que prejudicam a qualidade do PSF (Tabela 2).

Entre os elementos negativos destacados, cabe assinalar os seguintes: a) a maneira de atender dos profissionais, indicada como um aspecto crítico do serviço. Precisamente, foi enfatizado o

Tabela 2. O que prejudica a qualidade do PSF

\begin{tabular}{lc}
\hline \multicolumn{1}{c}{$\begin{array}{c}\text { Itens que prejudicam a } \\
\text { qualidade }\end{array}$} & $\begin{array}{c}\text { N. de usuários } \\
\text { que responderam }\end{array}$ \\
\hline $\begin{array}{l}\text { A maneira de atender dos } \\
\text { profissionais }\end{array}$ & 18 \\
$\begin{array}{l}\text { Demora no atendimento/ } \\
\text { Atraso no atendimento }\end{array}$ & 14 \\
Falta de fichas/falta de \\
atendimento
\end{tabular}


fato de o paciente "não ser examinado" e a insatisfação pelo manejo das relações interpessoais ("A consulta deveria ser mais demorada"; "Não explicam bem"; "Não informam bem”.); b) o longo tempo de espera no consultório e a demora para agendar a consulta ("Aqui é muito demorado para ser atendido"; "A gente chega às 7 h e sai às $12 \mathrm{~h}$ "; "Falta de fichas para o atendimento"; “As fichas são poucas").

\section{Discussão e conclusões}

Depois da análise dos dados, pode-se agora efetuar um trabalho de comparação entre as dimensões e indicadores da qualidade apontados por usuários, profissionais, gerentes e gestores, com o objetivo de identificar as áreas de consenso e as distâncias entre as três perspectivas de análise. Como se pode observar do Quadro 2, a "relação entre profissionais e pacientes" é um indicador de qualidade compartilhado por todos os informantes. É uma prioridade para profissionais e usuários e foi indicado como um dos aspectos mais importante para os gerentes e gestores.

$\mathrm{O}$ "ambiente" e a "estrutura física" onde se realizam as atividades do PSF, em coerência com outros estúdios internacionais, são considerados de fundamental importância para os profissionais. Gerentes e gestores identificaram esse item somente como terceira prioridade. No que diz respeito à estrutura do serviço, os pacientes se preocupam somente da "disponibilidade de medicamentos". É interessante notar que o principal elemento da qualidade apontado para gestores e gerentes é o "compromisso profissional"; esse indicador deve ser interpretado à luz do problema da elevada rotatividade dos médicos, retro analisado.
Outros aspectos que definem a qualidade da atenção no âmbito do PSF, evidenciado tanto pelos profissionais e como pelos gerentes e gestores é o desenvolvimento das "atividades de prevenção e promoção em saúde" e a "capacitação e atualização dos profissionais”. Os profissionais assinalam ainda a "adequação dos recursos humanos" que atuam no âmbito do PSF como forma de responder à grande demanda de atendimento da população. Finalmente, chama a atenção, como elemento associado à qualidade do cuidado, o item "ser atendido", evidenciado pelos pacientes como segunda prioridade. A valorização do simples fato de serem atendidos nos faz refletir sobre as difíceis experiências de acesso amadurecidas pelos usuários antes da implantação do PSF.

Estas últimas observações confirmam, ainda uma vez, como a qualidade em saúde não pode ser acertada somente por um único ator. Em outras palavras, como já assinalado por diferentes autores ${ }^{13,22,56,57}$, a qualidade não pode ser reduzida às dimensões privilegiadas somente pelos profissionais, nem às dimensões enfatizadas pelos gestores ou gerentes, e tampouco às dimensões percebidas pelos pacientes. De fato, os resultados deste estudo têm demonstrado como o ponto de vista dos pacientes possa resultar parcial e incompleto se for analisado sem considerar a visão de outros stakeholders. De acordo com diversos estudos internacionais ${ }^{25,52,58,59}$, a satisfação dos usuários, embora determinante, é insuficiente para avaliar a qualidade dos serviços de saúde, por diferentes razões: enorme desnível de informação entre pacientes e profissionais, inexistência de uma opinião do paciente, aceitação do paternalismo médico, etc.

No caso deste estudo, $85 \%$ têm avaliado positivamente a atenção recebida e 95\% têm expressado uma plena satisfação pela relação estabele-

Quadro 2. Aspectos aferentes à estrutura e ao processo que determinam a qualidade do atendimento na visão de Profissionais, Gerentes e Gestores, Usuários.

\begin{tabular}{|l|l|l|l|l|l|}
\hline \multicolumn{2}{|c|}{ Profissionais } & \multicolumn{1}{c|}{ Gerentes e Gestores } & \multicolumn{2}{c|}{ Usuários } \\
\hline \multicolumn{1}{|c|}{ Estrutura } & \multicolumn{1}{|c|}{ Processo } & Estrutura & \multicolumn{1}{c|}{ Processo } & \multicolumn{1}{c|}{ Estrutura } & \multicolumn{1}{c|}{ Processo } \\
\hline 2. Estrutura física & $\begin{array}{l}\text { 1. Relação } \\
\text { profissional-paciente }\end{array}$ & $\begin{array}{l}\text { 3. Estrutura } \\
\text { física }\end{array}$ & $\begin{array}{l}\text { 1. Compromisso } \\
\text { profissional }\end{array}$ & $\begin{array}{l}\text { 4. Disponibilidade } \\
\text { de remédios }\end{array}$ & $\begin{array}{l}\text { 1. Boa relação com } \\
\text { os profissionais }\end{array}$ \\
\hline $\begin{array}{l}\text { 4. Capacitação dos } \\
\text { profissionais }\end{array}$ & $\begin{array}{l}\text { 3. Promoção e } \\
\text { educação em saúde }\end{array}$ & $\begin{array}{l}\text { 5. Formação } \\
\text { profissional }\end{array}$ & $\begin{array}{l}\text { 2. Atenção dispensada } \\
\text { ao paciente }\end{array}$ & 2. Ser atendido \\
\hline $\begin{array}{l}\text { 6. Adequado } \\
\text { número de } \\
\text { profissionais }\end{array}$ & $\begin{array}{l}\text { 5. Compromisso } \\
\text { profissional }\end{array}$ & $\begin{array}{l}\text { 4. Atividades de } \\
\text { educação e promoção } \\
\text { da saúde }\end{array}$ & $\begin{array}{l}\text { 3. A boa atitude } \\
\text { do médico }\end{array}$ \\
\hline
\end{tabular}

OBS. - A numeração nos informa sobre a prioridade atribuída pelos informantes aos diversos aspectos. 
cida com enfermeiros e médicos; somente 15\% dos entrevistados têm manifestado algumas críticas sobre o atendimento. Ao contrário, os profissionais, mas também os gestores e gerentes conseguiram levantar alguns aspectos críticos inerentes à estrutura, à organização e aos processos de trabalho do PSF que poderiam ser trabalhados para melhorar a qualidade das práticas assistenciais.

Por outra parte, o estudo tem evidenciado também algumas dimensões da qualidade enfatizadas pelos pacientes, mas subestimadas pelos profissionais e gestores. Referimo-nos aos itens "disponibilidade de medicamentos nas unidades do PSF" e à possibilidade de "ser examinados pela equipe do PSF”. Também entre representante da oferta surgiram diversas avaliações. Para os gestores e gerentes, por exemplo, o "compromisso e a motivação” representam elementos essenciais da qualidade. Para os profissionais, ao invés disso, é prioritária a conformidade dos aspectos estruturais do serviço, tais como: o espaço físico, o equipamento e o ambiente de trabalho.
Como se pode notar, não há sempre coincidência entre os diversos olhares sobre o que è prioritário para garantir a qualidade do PSF. Outras investigações já tinham evidenciado a mesma discrepância nas percepções sobre a qualidade da atenção entre os diferentes atores ${ }^{38,47}$. É, portanto, importante começar a desenvolver estratégias de integração ou, pelo menos, de gradual aproximação entre os diversos pontos de vista. Esse é o grande desafio que os avaliadores deverão enfrentar nos próximos anos. Entretanto, o primeiro passo nessa direção é a promoção de práxis de avaliação inovadoras dentro das secretarias municipais de saúde e dentro das equipes de $\mathrm{PSF}^{10}$. Nessa linha, se recomenda aos gestores - que geralmente promovem e financiam estudos de avaliação - introduzir, no sistema de avaliação dos serviços, indicadores que possam medir os aspectos que definem a qualidade do cuidado do ponto de vista de usuários e profissionais. Somente dessa forma, o serviço pode realmente ser planejado e organizado para responder às necessidades de todos.

\section{Colaboradores}

Mauro Serapioni e Marcelo Gurgel Carlos da Silva participaram de todas as etapas de elaboração do artigo, desde o delineamento do estudo, a coleta e análise das informações até a redação final.

\section{Agradecimentos}

À Fundação Cearense de Apoio e Desenvolvimento Científico e Tecnológico (FUNCAP) e a Escola de Saúde Pública do Ceará (ESP/CE) pelo apoio financeiro. Pelo auxílio na pesquisa de campo os autores agradecem Antonio Silva Lima Neto, Valniza Araújo da Silva e Francileide Santana da Silva. Os autores agradecem também os preciosos pareceres dos avaliadores 


\section{Referências}

1. Vuori H. Quality assurance of health services. Copenaghen: WHO Regional Office for Europe; 1981. (Public Health in Europe, n. 16)

2. Organização Mundial da Saúde (OMS). Qualità dei servizi sanitari. Quaderni di sanità pubblica 1988; 57:4-36.

3. World Health Organization (WHO). Quality assessment and assurance in Primary Health Care. Programme Statement. Geneve: World Health Organization; 1989.

4. Vuori H. A qualidade da saúde. Divulg. saúde debate 1991; 3:17-24.

5. Franco LM, Silimperi DR, van Zanten TV, MacAulay C, Askov K, Marquez L. Sostenibilidad de la Calidad en la Atención de Salud: Institucionalización de la Garantía de la Calidad. Bethesda: USAID; 2004

6. Uchimora K, Bosi ML. Qualidade e subjetividade na avaliação de programas e serviços em saúde. Cad Saude Publica 2002; 18(6):151-169.

7. Illich I. Nemesi medica. L'espropriazione della salute, Milano: Mondadori; 1976.

8. Engel G. The need for a new medical model: a challenge for biomedicine. Science 1997; 196(4286): 129-136.

9. Eisenberg L, Kleinman A. The relevance of social science for medicine. Dordrecht: Reidel Publishing; 1981.

10. Serapioni M. Avaliação da qualidade em saúde. Contribuições para o delineamento de uma proposta muldimensional e correlacional. In: Bosi ML, Mercado-Martinez, FJ, organizadores. Avaliação qualitativa de programas de saúde. Enfoques emergentes. Petrópolis: Vozes; 2006. p. 207-227

11. Starfield B. Quality and outcomes framework: patient-centred? Lancet 2008; 372(9640):692-694.

12. Heath I, Rubinstein A, Stange KC, van Driel ML. Quality in primary health care: a multidimensional approach to complexity. BMJ 2009; 338:911-913.

13. Ranci Ortigosa E. La valutazione di qualità nei servizi sanitari. Milão: Angeli; 2000.

14. Donabedian A. La dimensión internacional de la evaluación y garantía de la calidad. Salud Publica Mex 1990; 32(2):113-117.

15. Guba E, Lincoln Y. Fourth Generation Evaluation. Newbury Park: Sage; 1989.

16. Patton MQ. Utilization-Focused Evaluation. The new century text. Thousand Oaks: Sage Publications; 1997.

17. Vasconcelos S, Bosi MLM, Pontes RJS et al. Avaliação e monitoramento da atenção básica no Estado do Ceará, Brasil: explorando concepções e experiências no nível central. Cad Saude Publica 2008; 24(12):2891-2900.

18. Felisberto E. Da teoria à formulação de uma Política Nacional de Avaliação em Saúde: reabrindo o debate. Cien Saude Colet 2006; 11(3):553-556.

19. Donabedian A. The definition of Quality and Approaches to its Assessment. Vol. I. Ann Arbour: Health Administration Press, University of Michigan; 1980.

20. Donadedian Avedis. La qualità dell'assistenza sanitaria. Roma: Nuova Italia Scientifica; 1989.
21. Øvretveit J. La qualità nel servizio sanitario. Napoli: Edizione ISES; 1996

22. Øvretveit J. Evaluating health interventions. Buckingham: Open University Press; 1998.

23. Souza H. Saúde da Família: desafios e conquistas. In: Negri B, Viana AL, organizadores. O sistema Único de Saúde em dez anos de desafio. São Paulo: SOBRAVIME/CELAG; 2002.

24. Heimann LS, Mendonça MH. A trajetória da atenção básica em saúde e do programa de Saúde da família no SUS: uma busca de identidade. In: Lima NT, Gershman S, Edler FC, organizadores. Saúde e democracia. História e perspectivas do SUS. Rio de Janeiro: Fiocruz; 2005. p. 481-502.

25. Conill EM. A historical and conceptual model for Primary Health Care: challenges for the organization of primary care and the Family Health Strategy in large Brazilian cities. Cad Saude Publica 2008; 24(Supl. 1):7-16.

26. Bodstein R. Processo decisório e avaliação em saúde: ampliando o debate sobre o Programa Saúde da Família. Cien Saude Colet 2009; 14(Supl.1):13361338.

27. Brasil. Ministério da Saúde (MS). Avaliação da Implantação e Funcionamento do Programa de Saúde da Família - PSF. Brasília: Ministério da Saúde (MS); 1999.

28. Brasil. Ministério da Saúde (MS). Avaliação da implementação do Programa Saúde da Família em dez grandes centros urbanos. Brasília: Ministério da Saúde (MS); 2004.

29. Brasil. Ministério da Saúde (MS). Avaliação da Atenção Básica em Saúde: caminhos da institucionalização. Brasília: Ministério da Saúde (MS); 2005.

30. Facchini LA. Desempenho do PSF no Sul e no Nordeste do Brasil: avaliação institucional e epidemiológica da Atenção Básica à Saúde. Cien Saude Colet 2006, 11(3):6669-6681.

31. Viana ALA. Modelos de atenção básica nos grandes municípios paulistas: efetividade, eficácia, sustentabilidade e governabilidade. Cien Saude Colet 2006; 11(3):557-606.

32. Ibañez N, Rocha JSY, Castro PC, Ribeiro MCSA, Forster AC, Novaes MHD, Viana ALd'A. Avaliação do desempenho da atenção básica no Estado de São Paulo. Cien Saude Colet 2006; 11(3):683-703.

33. Henrique F, Calvo MCM. Avaliação do Programa Saúde da Família nos municípios do Estado de Santas Cararina, Brasil. Cad Saude Publica 2008; 24(4): 809-819.

34. Costa GD, Cotta RMM, Reis JR, Siqueira-Batista R, Gomes AP, Franceschini SCC. Avaliação do cuidado à saúde da gestante no contexto do Programa Saúde da Família. Cien Saude Colet 2009; 14(Supl. 1): 1347-1357.

35. Nagahama EEI. Avaliação da implantação de serviços de saúde reprodutiva no Município de Maringá, Paraná, Brasil. Cad Saude Publica 2009; 25(Supl. 2):S279-S290.

36. Viana ALA, Pierantoni CR, Heimann LS, Lima LD, Oliveira RG. Indicadores de monitoramento da implementação do PSF em grandes centros urbanos. Brasília: Ministério da Saúde (MS); 2002. 
37. Escorel S, Giovanella L, Mendonça MH, Magalhaes R, Senna M. Avaliação da implementação do programa de saúde da família em dez grandes centros urbanos. Brasília: Ministério da Saúde (MS); 2002.

38. Almeida C, Macinko, J. Validação de uma metodologia de avaliação rápida das características organizacionais e do desempenho dos serviços de atenção básica do Sistema Único de Saúde (SUS) em nivel local. Brasília: Organização Pan-Americana da Saúde/Ministério da Saúde (MS); 2006.

39. Almeida PF, Giovanella L. Assessment of Primary Health care in Brazil: mapping and analysis of research conducted and/or financed by the Ministry of Health from 2000 to 2006. Cad Saude Publica 2008 24(8):1727-1742.

40. Contandriopoulos AP. Avaliando a institucionalização da avaliação. Cien Saude Colet 2006; 11(3):705711.

41. Yin RK. Case Study Research. Design and Methods. Fourth Edition. Thousand Oaks: Sage Publications; 1994.

42. Bardin L. Análise de conteúdo. Lisboa: Edições 70; 1995.

43. Quivy R, Campenhoudt LV. Manual de Investigación en Ciencias Sociales. Limusa Noriega: México; 1998.

44. Hopkins A, Gabbay Y, Neuberger Y. Role of users of health care in achieving a quality service. Qual Health Care 1994; 3(4):203-209.

45. Campbell SM, Roland MO, Buetow SA. Defining quality of care. Social Science and Medicine 2000; 51(11):1611-1625.

46. Jackson J, Chamberlin J, Kroenke K. Predictors of patient satisfaction. Social Science and Medicine 2001, 52(4):609-620

47. Ferreira PL. Avaliação dos doentes de cuidado primário. Aspectos da clínica geral mais importantes para os doentes. Revista Port. Clínica Geral 2001; 17:15-45.

48. Ronzani TM, Silva CM. O Programa Saúde da Família segundo profissionais de saúde, gestores e usuários. Cien Saude Colet 2008; 13(1):23-34.

49. Mazza L. Il concetto di soddisfazione. Salute e Territorio $1998 ; 107: 30-38$.

50. Atkinson S, Medeiros R. Explanatory models of influences on the construction and expression of user satisfaction. Soc Sci Med 2009; 68(11):2089-2096.
51. Vaitsman J, Andrade BGR. Satisfação e responsividade: formas de medir a qualidade e a humanização da assistência à saúde. Cien Saude Colet 2005; 10(3):599-613.

52. Williams B. Patient Satisfaction: A Valid Concept? Soc Sci Med 1994; 38(4):509-516.

53. Avis M. Questioning patient satisfaction: an empirical investigation in two outpatient clinics. Soc Sci Med 1997; 44(1):85-92.

54. Altieri L. Valutazione della qualità/qualità della valutazione. Problemi metodologici della ricerca valutativa in sanità. In: Altieri L, Luison L, organizadores. Qualità e strumenti sociologici. Milano: Angeli; 1997. p. 93-114

55. Corposanto C. Metodologia e tecniche non intrusive nella ricerca sociale. Milão: Angeli; 2004.

56. Fitzpatrick R. Measurement of patient satisfaction. In: Hopkins A, Costain D, organizadores. Measuring the Outcome of Medical Care. London: Royal College of Phisicians of London \& King's Fund; 1990.

57. Giarelli G. Le connessioni possibili: i sistemi di miglioramento della qualità. In: Cipolla C, Giarelli G, Altieri L, organizadores. Valutare la qualità in sanità. Milão: Angeli; 2002. p. 430-437.

58. Hopkins A. Measuring the Quality of Medical Care. London: Royal College of Phisicians of London; 1990.

59. Robertson A. Definire e valutare la qualità nei servizi socio-sanitari. In: Bertin G, organizador. Valutazione e sapere sociologico. Milano: Angeli; 1995. p. 105-125

Artigo apresentado em 30/07/2010

Aprovado em 29/11/2010

Versão final apresentada em 05/12/2010 